\title{
Classical Computation of Elliptic Flow at Large Transverse Momentum
}

\author{
Derek Teaney \\ Physics Department, Brookhaven National Laboratory, Upton, N.Y. 11973, U.S.A. \\ Raju Venugopalan \\ Physics Department and RIKEN-BNL Research Center, \\ Brookhaven National Laboratory, Upton, N.Y. 11973, U.S.A.
}

(Dated: November 1, 2018)

We compute the contribution of classical fields to the second Fourier coefficient $\left(v_{2}\right)$ of the azimuthal gluon distribution at large transverse momentum in heavy ion collisions. We find that the classical contribution to the flow alone cannot account for the experimentally observed behavior of $v_{2}\left(p_{t}\right)$ at large transverse momentum $p_{t}$.

\section{INTRODUCTION}

The azimuthal anisotropy of the particles produced in heavy ion collisions is quantified by the Elliptic flow parameter $v 2$. It is defined to be the second Fourier moment of the azimuthal distribution,

$$
v_{2}=\langle\cos (2 \phi)\rangle \equiv \frac{\int_{-\pi}^{\pi} d \phi \cos (2 \phi) \int p_{t} d p_{t} \frac{d^{3} N}{d y p_{t} d p_{t} d \phi}}{\int_{-\pi}^{\pi} d \phi \int p_{t} d p_{t} \frac{d^{3} N}{d y p_{t} d p_{t} d \phi}} .
$$

The RHIC (Relativistic Heavy Ion Collider) experiments have measured the centrality dependence of $v_{2}$ and find a value for $v_{2}$ [1] that is significantly larger for non-central collisions than previous measurements at lower energies [2]. Hydrodynamic models do a reasonable job of describing the centrality and mass dependence of $v_{2}$ [3, 4]. The RHIC experiments have also measured $v_{2}\left(p_{t}\right)$, which is defined to be

$$
v_{2}\left(p_{t}\right)=\frac{\int_{-\pi}^{\pi} d \phi \cos (2 \phi) \frac{d^{3} N}{d y p_{t} d p_{t} d \phi}}{\int_{-\pi}^{\pi} d \phi \frac{d^{3} N}{d y p_{t} d p_{t} d \phi}} .
$$

The data [1, 5] show that, for a wide range of centralities, $v_{2}\left(p_{t}\right)$ rises up to $1.5 \mathrm{GeV}$, flattens and then remains flat up to the highest transverse momentum measured $p_{t} \approx 6 \mathrm{GeV}$. For 
peripheral collisions (specifically $34 \%-85 \%$ central collisions), $v_{2}\left(p_{t}\right)$ can be as large as $25 \%$ at $p_{t} \sim 3 \mathrm{GeV}$.

Hydrodynamic models describe the initial rise in $v_{2}\left(p_{t}\right)$ but overpredict the data at large $p_{t}$. The models predict a continued rise except perhaps in a Blast wave parametrization with extreme assumptions [6]. Partonic descriptions which account for the quenching of high $p_{t}$ "jets" as they traverse the hot QCD matter do not describe the data either [7]-unless unusually large partonic cross-sections are assumed [8].

In this note, we discuss the computation of $v_{2}$ in the Color Glass Condensate (CGC) approach to nuclear collisions [9, 10, 12]. At very high energies, the density of partons per unit area in the colliding nuclei becomes very large and saturates at a scale $Q_{s} \gg \Lambda_{Q C D}$ 13. For RHIC, estimates give $Q_{s} \sim 1-2 \mathrm{GeV}$ [12, 14, 10, 16]. The typical occupation number of partons is $1 / \alpha_{s}\left(Q_{s}\right)>1$. Thus classical methods can be used to describe nuclear collisions at high energies. The CGC approach has been used to describe successfully various aspects of the bulk properties of charged hadrons in heavy ion collisions, including the multiplicity [12], the centrality dependence of the multiplicity [16], the energy and rapidity dependence [17] and the $p_{t}$ dependence of inclusive hadron spectra [18].

Elliptic flow, as defined in Eq. 1, is dominated by contributions from small momenta. In the classical approach, this involves computing diagrams to all orders in $Q_{s} / p_{t}$. This computation is very difficult to perform analytically. It can be computed numerically [20]. However, when the transverse momenta is large, $p_{t} \gg Q_{s}$, the classical equations can be linearized and the contribution to $v_{2}\left(p_{t}\right)$ can then be computed analytically. This computation is performed in this paper. We find that the contribution to $v_{2}\left(p_{t}\right)$ from classical gluon fields at large momenta is small and differs significantly from the measured distribution at high $p_{t}$. The origin of the empirically observed behavior of $v_{2}\left(p_{t}\right)$, at large $p_{t}$, must arise from a source outside the classical perturbative expansion. Interestingly, even though the contribution from high momenta is small, this contribution is generated at very early times.

The paper is organized as follows. In the following section, we set up the problem of computing the classical gluon distribution at large transverse momentum. In section 3, we perform the computation. A brief final section summarizes our results. Some details of the computation are relegated to an appendix. 


\section{THE CLASSICAL GLUON DISTRIBUTION}

Within the framework of the McLerran-Venugopalan model, Kovner, McLerran, and Weigert (KMW) [10] (see also Refs. [11] and [14]) computed the classical spectrum of hard gluons (with $p_{t}>Q_{s}$ ) radiated from a collision of two infinitely large nuclei with uniform color charge distributions. In order to calculate the azimuthal distribution of the radiated gluons, we generalize this work to a finite nucleus with a non-uniform color charge distribution. Gradients in the charge distribution then give rise to space-momentum correlations of the radiated gluons. The typical momentum scale of the produced gluons is $\sim Q_{s}\left(x_{t}\right)$. A typical spatial gradient is $\sim 1 / R$. Therefore, the typical space-momentum correlation is of order $\sim\left(Q_{s} R\right)^{-1}$. The final expression for $v_{2}$ is actually of order $\sim\left(Q_{s} R\right)^{-2}$ since elliptic flow responds to the quadrupole moment of the color charge distribution.

We first review the perturbative solutions to the classical Yang Mills equations in order to establish the notation. In the McLerran-Venugopalan model, two nuclei (A and B) collide at very high energies. The valence partons generate classical fields at very small Bjorken $x$. The subsequent evolution of these classical fields is determined by the equations of motion. Therefore, the solution to the equations of motion is a functional of the valence color charge density (per unit area per unit rapidity) in nuclei A and B $-\rho_{A}\left(x_{t}\right)$ and $\rho_{B}\left(x_{t}\right)$, respectively. The expectation of any quantity $O$ is first expressed in terms of the classical solution and then averaged over all possible valence charge distributions with a weight function. In the McLerran-Venugopalan model, a Gaussian ansatz is taken for the weight function. Specifically, for a functional $\mathrm{O}\left(\rho_{A}, \rho_{B}\right)$ we have the following average:

$$
\langle O\rangle_{\rho} \equiv \int\left[D \rho_{A}\right]\left[D \rho_{B}\right] O\left(\rho_{A}, \rho_{B}\right) \exp \left(-\int d^{2} x_{t} \frac{\operatorname{Tr}\left(\rho_{A}^{2}\left(x_{t}\right)\right)}{\mu_{A}^{2}\left(x_{t}\right)}-\int d^{2} y_{t} \frac{\operatorname{Tr}\left(\rho_{B}^{2}\left(y_{t}\right)\right)}{\mu_{B}^{2}\left(y_{t}\right)}\right) .
$$

At large transverse momentum, the density of charges is small and the field strength is weak. Therefore the classical Yang-Mills equations can be linearized and the solution can be expressed as a functional of the sources in closed form. The final distribution of gluons can then be related to the averages of the gluon field. The analysis of [10] shows that the distribution of radiated gluons is given by

$$
\begin{aligned}
\frac{d N}{d y d^{2} k_{t}} \propto & \int d^{2} x_{t} d^{2} y_{t} e^{i k_{t} \cdot x_{t}} e^{-i k_{t} \cdot y_{t}} \operatorname{Tr}\left\langle\left[\partial^{i} \phi_{A}, \partial^{k} \phi_{B}\right]\left(x_{t}\right)\left[\partial^{j} \phi_{A}, \partial^{l} \phi_{B}\right]^{\dagger}\left(y_{t}\right)\right\rangle_{\rho} \\
& \times\left(\delta^{i j} \delta^{k l}+\epsilon^{i j} \epsilon^{k l}\right),
\end{aligned}
$$


where $\phi_{A}\left(\phi_{B}\right)$ is the 2-d Coulomb potential associated with the color charge distribution $\rho_{A}\left(\rho_{B}\right)$,

$$
-\nabla_{T}^{2} \phi_{A}\left(x_{T}\right)=g \rho_{A}
$$

In what follows, we evaluate the average over $\rho$ and and perform the necessary Fourier transforms in order to determine the gluon distribution to order $\sim\left(Q_{s} R\right)^{-2}$. With this gluon distribution, we determine the azimuthal anisotropy, $v_{2}\left(p_{t}\right)$.

\section{COMPUTATION OF $v_{2}(k)$ FOR $k>Q_{s}$}

The averages over the color sources in Eq. A can be performed most simply in Fourier space. Below, we adopt the convention that repeated spatial indices are integrated over and repeated Fourier indices are integrated over with the appropriate two dimensional measure, $\int \frac{d^{2} k}{(2 \pi)^{2}}$. In Fourier space, the average of $O\left(\rho_{A}, \rho_{B}\right)$ is then

$$
\langle O\rangle_{\rho}=\int\left[D \tilde{\rho_{A}}\right]\left[D \tilde{\rho_{B}}\right] O\left(\rho_{A}, \rho_{B}\right) \exp \left(-\operatorname{Tr}\left(\tilde{\rho}_{A}\right)_{k}^{*}\left(C_{A}\right)_{k k^{\prime}}\left(\tilde{\rho}_{A}\right)_{k^{\prime}}-\operatorname{Tr}\left(\tilde{\rho}_{B}\right)_{k}^{*}\left(C_{B}\right)_{k k^{\prime}}\left(\tilde{\rho}_{B}\right)_{k^{\prime}}\right)
$$

where $\left(\tilde{\rho}_{A}\right)_{k}=e^{i k \cdot x} \rho_{A}(x)$ is the Fourier transform of $\rho_{A}(x)$ and $\left(C_{A}\right)_{k k^{\prime}}$ is given by

$$
\left(C_{A}\right)_{k k^{\prime}}=e^{i k \cdot x} \frac{\delta_{x x^{\prime}}}{\mu_{A}^{2}(x)} e^{-i k^{\prime} \cdot x^{\prime}}
$$

Then pairwise correlations for nucleus A are given by

$$
\left\langle\left(\tilde{\rho_{A}}\right)_{k}^{*}\left(\tilde{\rho_{A}}\right)_{l}\right\rangle=\left(C_{A}^{-1}\right)_{k l}=e^{i(k-l) \cdot x} \mu_{A}^{2}(x) \equiv \tilde{\mu^{2}}{ }_{A}(k-l)
$$

Fourier transforming each of the $\phi$ 's, the gluon distribution of Eq. A becomes

$$
\frac{d N}{d y d^{2} k} \propto e^{i k \cdot x_{t}} e^{i l_{1} \cdot x_{t}} e^{i l_{2} \cdot x_{t}} e^{-i k \cdot y_{t}} e^{-i l_{3} \cdot y_{t}} e^{-i l_{4} \cdot y_{t}}\left\langle\frac{l_{1}}{l_{1}^{2}}\left(\tilde{\rho}_{A}\right)_{l_{1}} \frac{l_{2}}{l_{2}^{2}}\left(\tilde{\rho}_{B}\right)_{l_{2}} \frac{l_{3}}{l_{3}^{2}}\left(\tilde{\rho}_{A}\right)_{l_{3}}^{*} \frac{l_{4}}{l_{4}^{2}}\left(\tilde{\rho}_{B}\right)_{l_{4}}^{*}\right\rangle_{\rho}
$$

Contracting the $\rho_{A}$ variables and the $\rho_{B}$ variables we find

$$
\frac{d N}{d y d^{2} k} \propto \frac{\delta^{i j} \delta^{k l}+\epsilon^{i j} \epsilon^{k l}}{l_{1}^{2} l_{3}^{2}\left(l_{1}+k\right)^{2}\left(l_{3}+k\right)^{2}} l_{1}^{i} l_{3}^{k}\left(l_{1}+k\right)^{j}\left(l_{3}+k\right)^{l}\left(C_{A}^{-1}\right)_{l_{1} l_{3}}^{*}\left(C_{B}^{-1}\right)_{\left(l_{1}+k\right)\left(l_{3}+k\right)} .
$$

Specializing now to the case where the target and projectile are identical, restoring the implicit integration, and using $\left(C_{A}^{-1}\right)_{k l}=\left(C_{B}^{-1}\right)_{k l}=\tilde{\mu^{2}}(k-l)$ we obtain

$$
\frac{d N}{d y d^{2} k} \propto \iint \frac{d^{2} l_{1}}{(2 \pi)^{2}} \frac{d^{2} l_{3}}{(2 \pi)^{2}} \frac{\delta^{i j} \delta^{k l}+\epsilon^{i j} \epsilon^{k} l_{3}^{2}\left(l_{1}+k\right)^{2}\left(l_{3}+k\right)^{2}}{l_{1}^{i}} l_{3}^{k}\left(l_{1}+k\right)^{j}\left(l_{3}+k\right)^{l}\left|\tilde{\mu}^{2}\left(l_{1}-l_{3}\right)\right|^{2} .
$$


For the purposes of illustration, we consider a Gaussian model charge distribution [19]

$$
\begin{aligned}
\mu^{2}(\vec{x}) & =\frac{Q^{2}}{2 \pi R_{x} R_{y}} e^{-\frac{x^{2}}{2 R_{x}^{2}}-\frac{y^{2}}{2 R_{y}^{2}}} \\
\tilde{\mu^{2}}(\vec{k}) & =Q^{2} e^{-\frac{1}{2} k_{x}^{2} R_{x}^{2}-\frac{1}{2} k_{y}^{2} R_{y}^{2}}
\end{aligned}
$$

where $R_{x}$ and $R_{y}$ denote the transverse spatial extent (in the $x$ and $y$ direction) of the overlap region of the two nuclei and $Q^{2}$ is the net color charge squared in the overlap region.

For this model distribution, we see that $\left|\tilde{\mu}^{2}\left(l_{1}-l_{3}\right)\right|^{2}$ is a sharply falling function. Thus $\left|\tilde{\mu}^{2}\left(l_{1}-l_{3}\right)\right|^{2}$ is approximately a delta function. Indeed, when $\left|\tilde{\mu}^{2}\left(l_{1}-l_{3}\right)\right|^{2}$ is a delta function Eq. 11 reduces to the result of [10] for the gluon distribution. We therefore approximate the integral over $\mu^{4}$ as

$$
\begin{aligned}
\int \frac{d^{2} z}{(2 \pi)^{2}}\left|\tilde{\mu}^{2}(z)\right|^{2} f(z) \approx & \left(\tilde{\mu}^{4}\right)^{(0)} f(0)+\frac{1}{2}\left(\tilde{\mu}^{4}\right)_{i j}^{(2)}\left(\frac{\partial^{2} f}{\partial z_{i} \partial z_{j}}-\frac{\delta^{i j}}{2} \frac{\partial^{2} f}{\partial_{z_{l}} \partial_{z_{l}}}\right)_{z=0} \\
& +\frac{1}{2}\left(\tilde{\mu}^{4}\right)_{i j}^{(2)}\left(\frac{\delta^{i j}}{2} \frac{\partial^{2} f}{\partial_{z_{l}} \partial_{z_{l}}}\right)_{z=0} .
\end{aligned}
$$

In writing the above equation we have assumed that the charged distribution $\left|\tilde{\mu}^{2}(z)\right|^{2}$ is symmetric in $z$ and defined the moments of this distribution as

$$
\begin{aligned}
\left(\tilde{\mu}^{4}\right)^{(0)} & \equiv \int \frac{d^{2} z}{(2 \pi)^{2}}\left|\tilde{\mu}^{2}(z)\right|^{2} \\
\left(\tilde{\mu}^{4}\right)_{i j}^{(2)} & \equiv \int \frac{d^{2} z}{(2 \pi)^{2}} z_{i} z_{j}\left|\tilde{\mu}^{2}(z)\right|^{2} .
\end{aligned}
$$

For the problem at hand, we have

$$
\frac{d N}{d y d^{2} k} \propto \int \frac{d^{2} z}{(2 \pi)^{2}}\left|\tilde{\mu}^{2}(z)\right|^{2} f(k, z)
$$

where $f(k, z)$ is given by

$$
f(k, z) \equiv \int \frac{d^{2} p}{(2 \pi)^{2}} \frac{\delta^{i j} \delta^{k l}+\epsilon^{i j} \epsilon^{k l}}{p^{2}(p-z)^{2}(p+k)^{2}(p-z+k)^{2}} p^{i}(p-z)^{k}(p+k)^{j}(p-z+k)^{l} .
$$

We may write down the general form of the second derivatives of $f(k, z)$

$$
\begin{aligned}
&\left(\frac{\partial^{2} f(k, z)}{\partial z_{i} \partial z_{j}}-\frac{\delta^{i j}}{2} \frac{\partial^{2} f(k, z)}{\partial_{z_{l}} \partial_{z_{l}}}\right)_{z=0} \equiv f^{(2)}\left(k^{2}\right)\left(\frac{k_{i} k_{j}}{k^{2}}-\frac{\delta^{i j}}{2}\right) \\
&\left(\frac{\partial^{2} f(k, z)}{\partial_{z_{l}} \partial_{z_{l}}}\right)_{z=0} \equiv g^{(2)}\left(k^{2}\right) .
\end{aligned}
$$


Now the elliptic flow parameter $v_{2}$ is given by

$$
v_{2}(y, k) \equiv \frac{\int \frac{d \phi}{2 \pi} \cos (2 \phi) \frac{d N}{d y k d k d \phi}}{\int \frac{d \phi}{2 \pi} \frac{d N}{d y k d k d \phi}} .
$$

Substituting Eq. 14 and Eq. 19 into this definition of $v_{2}$, we obtain to leading order in $\left(Q_{s} R\right)^{-1}$

$$
v_{2}(y, k)=\frac{\left(\mu^{4}\right)_{x x}^{(2)}-\left(\mu^{4}\right)_{y y}^{(2)}}{8\left(\mu^{4}\right)^{(0)}} \frac{f^{(2)}\left(k^{2}\right)}{f(k, z=0)} .
$$

$f(k, z=0)$ and $f^{(2)}\left(k^{2}\right)$ are calculated in appendix A. The results are logarithmically divergent. As discussed in [10], this is a consequence of the weak field expansion; higher order non-linear corrections cut off the behavior in the infra-red at a scale $\alpha_{s} \mu$. To leading $\log$ accuracy we find

$$
\begin{aligned}
f(k, z=0) & \approx \frac{1}{(2 \pi)} \frac{1}{k^{2}} \log \left(\frac{k^{2}}{\left(\alpha_{s} \mu\right)^{2}}\right) \\
f^{(2)}\left(k^{2}\right) & \approx \frac{1}{(2 \pi)} \frac{4}{k^{4}} \log \left(\frac{k^{2}}{\left(\alpha_{s} \mu\right)^{2}}\right) .
\end{aligned}
$$

With these expressions we obtain our final result for $v_{2}$

$$
v_{2}(y, k) \approx \frac{1}{2 k^{2}} \frac{\left(\mu^{4}\right)_{x x}^{(2)}-\left(\mu^{4}\right)_{y y}^{(2)}}{\left(\mu^{4}\right)^{(0)}}
$$

It is remarkable (see the appendix) that there are no power or even logarithmic divergences from the ratios of integrals in our expression for $v_{2}$. For the Gaussian distribution of Eq. 12 the moments of the charge distribution are easily calculated. The resulting elliptic flow is

$$
v_{2}(y, k) \approx \frac{1}{4 k^{2}}\left(\frac{1}{R_{x}^{2}}-\frac{1}{R_{y}^{2}}\right) .
$$

The contribution of momenta $k>Q_{s}$ to the integrated $v_{2}$ (Eq. 1) is

$$
v_{2}=\frac{\int_{Q_{s}}^{\infty} k d k v_{2}(k) \frac{d N}{d^{2} k}}{\int_{Q_{s}}^{\infty} d^{2} k \frac{d N}{d^{2} k}} \propto \frac{1}{Q_{s}^{2}}\left(\frac{1}{R_{x}^{2}}-\frac{1}{R_{y}^{2}}\right) .
$$

For realistic values of $Q_{s} \sim 1 \mathrm{GeV}$ for $\mathrm{RHIC}$ and $R_{x} \sim 3 \mathrm{fm}$ for a peripheral collision, this contribution is a fraction of a percent. The significant contribution of classical fields to $v_{2}$ comes from momenta $k<Q_{s}$. Interestingly, our results suggest that $v_{2}$ for $k>Q_{s}$ is generated very early. The spatial and temporal components decouple already at proper time $\tau=0$. The temporal contributions are Bessel functions $J_{0}(k \tau)$ and $J_{1}(k \tau)$ which can be linearized into plane waves for times $\tau<1 / Q_{s}$ when $k>>Q_{s}$. 


\section{SUMMARY}

In this paper, we have computed analytically the contribution to the azimuthal anisotropy $\left(v_{2}(k)\right)$ from classical fields at large transverse momenta, $k^{2}>Q_{s}^{2}$. We find that the azimuthal anisotropy has the simple form shown in Eq. 26 for a Gaussian charge distribution. Clearly, this behavior disagrees with the data and another mechanism must be found to explain the large momentum behavior. Non-flow azimuthal correlations may provide a natural explanation [21]. An interesting feature of our result is that the temporal dependence of the perturbative classical fields decouples already at proper time $\tau=0$ [10]. Thus as Eq. 团 suggests, the momentum anisotropy is generated at very early times in the collision. This analysis may thus explain why (in numerical simulations of the Yang-Mills equations [20]) a significant anisotropy is seen at early times after the collision.

\section{ACKNOWLEDGEMENTS}

We would like to thank D. Kharzeev, Y. Kovchegov, A. Krasnitz, L. McLerran, Y. Nara and K. Tuchin for useful discussions. R.V and D.T.'s work was supported by DOE Contract No. DE-AC02-98CH10886. R.V would also like to acknowledge RIKEN-BNL for support.

\section{APPENDIX A: EVALUATION OF INTEGRALS}

To complete the evaluation of $v_{2}$ we expand Eq. 17 as a function of $z$ and extract the coefficients of the symmetric traceless tensor structures. We find after some algebra

$$
\begin{aligned}
f(k, z=0) & =\int \frac{d^{2} p}{(2 \pi)^{2}} \frac{1}{p^{2}(k+p)^{2}} \\
f^{(2)}\left(k^{2}\right)\left(\frac{k_{i} k_{j}}{k^{2}}-\frac{\delta_{i j}}{2}\right) & =\left(I^{1}\right)_{i j}+\left(I^{2}\right)_{i j}+\left(I^{3}\right)_{i j}
\end{aligned}
$$

where the integrals are given by

$$
\begin{aligned}
\left(I^{1}\right)_{i j} & =\int \frac{d^{2} p}{(2 \pi)^{2}} \frac{2}{p^{2}(k+p)^{6}}\left(k_{i} k_{j}-k^{2} \frac{\delta_{i j}}{2}\right) \\
\left(I^{2}\right)_{i j} & =\int \frac{d^{2} p}{(2 \pi)^{2}} \frac{4}{p^{2}(k+p)^{6}}\left(\frac{k_{i} p_{j}+p_{i} k_{j}}{2}-p \cdot k \frac{\delta_{i j}}{2}\right) \\
\left(I^{3}\right)_{i j} & =\int \frac{d^{2} p}{(2 \pi)^{2}}\left(\frac{2}{p^{2}(k+p)^{6}}+\frac{2}{p^{6}(p+k)^{2}}\right)\left(p_{i} p_{j}-p^{2} \frac{\delta_{i j}}{2}\right) .
\end{aligned}
$$


$f(k, z=0)$ is logarithmically divergent at $p=0$ and $\vec{p}=-\vec{k}$. It is identical to the expression obtained previously in [10, 14]. As discussed there and shown explicitly in Ref. [12] this infra-red divergence is an artifact of the weak field expansion. To leading log accuracy we find

$$
f(k, z=0) \approx \frac{1}{(2 \pi)} \frac{1}{k^{2}} \log \left(\frac{k^{2}}{\left(\alpha_{s} \mu\right)^{2}}\right) .
$$

Consider now the sum of the integrals in Eqs. A5. While each of these individually have power divergences, taken together, the result is again only logarithmically divergent. Note, as previously, the integrals are divergent at $p=0$ and $\vec{p}=-\vec{k}$ and one obtains logarithmic contributions from both regions to the final result. For illustration, consider the contribution to the sum from the divergence around $p=0 .\left(I^{1}\right)_{i j}$ is logarithmically divergent, $\left(I^{2}\right)_{i j}$ is finite, and $\left(I^{3}\right)_{i j}$ appears power divergent. However, $\left(I^{3}\right)_{i j}$ is actually only logarithmically divergent upon integration over the angle between $p$ and $k$. Contracting both sides of Eq. A2 with $2\left(\frac{k_{i} k_{j}}{k^{2}}-\frac{\delta_{i j}}{2}\right)$ and keeping only logarithmically divergent terms we obtain the contribution of the $p=0$ divergence to $\left(I^{1}\right)_{i j}$ and $\left(I^{3}\right)_{i j}$ respectively to be

$$
\begin{aligned}
& 2\left(I_{1}\right)_{i j}\left(\frac{k^{i} k^{j}}{k^{2}}-\frac{\delta^{i j}}{2}\right)_{|\mathrm{p}| \approx \alpha_{\mathrm{s}} \mu}=k^{2} \int \frac{d^{2} p}{(2 \pi)^{2}} \frac{2}{p^{2}(k+p)^{6}} \approx \frac{1}{(2 \pi)} \frac{1}{k^{4}} \log \left(\frac{k^{2}}{\left(\alpha_{s} \mu\right)^{2}}\right) \\
& 2\left(I_{3}\right)_{i j}\left(\frac{k^{i} k^{j}}{k^{2}}-\frac{\delta^{i j}}{2}\right)_{|\mathrm{p}| \approx \alpha_{\mathrm{s}} \mu}=\int \frac{d^{2} p}{(2 \pi)^{2}} \frac{2 p^{2} \cos \left(2 \phi_{p k}\right)}{p^{6}(k+p)^{2}} \approx \frac{1}{(2 \pi)} \frac{1}{k^{4}} \log \left(\frac{k^{2}}{\left(\alpha_{s} \mu\right)^{2}}\right)
\end{aligned}
$$

where $\phi_{p k}$ is the angle between $p$ and $k$. A detailed analysis shows that the contribution to the sum $\left(I^{1}\right)_{i j}+\left(I^{2}\right)_{i j}+\left(I^{3}\right)_{i j}$ from the logarithmic divergence around $\vec{p}=-\vec{k}$ is identical to the sum of the divergent pieces in Eqs. A7.

Substituting the results of the above analysis into the expression for $f^{(2)}\left(k^{2}\right)$ we find

$$
f^{(2)}\left(k^{2}\right)=\frac{1}{(2 \pi)} \frac{4}{k^{4}} \log \left(\frac{k^{2}}{\left(\alpha_{s} \mu\right)^{2}}\right) .
$$

With these expressions for $f(k, z=0)$ and $f^{(2)}\left(k^{2}\right)$, the final result for $v_{2}$ given in Eq. 25 follows.

[1] STAR Collaboration, K.H. Ackermann et al., Phys. Rev. Lett. 86, 402 (2001); STAR Collaboration, C. Adler et al., Phys. Rev. Lett. 87, 182301 (2001).

[2] NA49 Collaboration, H. Appelshäuser et al., Phys. Rev. Lett. 80, 4136 (1998); A.M. Poskanzer and S.A. Voloshin for the NA49 Collaboration, Nucl. Phys. A661, 341c (1999). 
[3] D. Teaney, J. Lauret, and E.V. Shuryak, Phys. Rev. Lett. 86, 4783 (2001); D. Teaney, J. Lauret, and E.V. Shuryak, nucl-th/0110037.

[4] P.F. Kolb, P.Huovinen, U. Heinz, H. Heiselberg, Phys. Lett. B 500, 232 (2001); P. Huovinen, P.F. Kolb, U. Heinz, H. Heiselberg, Phys. Lett. B 503, 58 (2001).

[5] R.J.M. Snellings for the STAR Collaboration, Nucl. Phys. A698, 611c (2002).

[6] P.J. Siemens and J.O. Rasmussen, Phys. Rev. Lett. 42, 880 (1979); E. Schnederman, J. Sollfrank, U.Heinz, Phys. Rev. C48, 2462 (1993); P. Huovinen, P.F. Kolb, U. Heinz, Nucl. Phys. A698, 475 (2002).

[7] M. Gyulassy, I. Vitev, X.N. Wang, Phys. Rev. Lett. 86, 2537 (2001).

[8] D. Molnar and M. Gyulassy, Nucl. Phys. A697, 495 (2002); D. Molnar and M. Gyulassy, Nucl. Phys. A698, 379 (2002).

[9] L. McLerran and R. Venugopalan, Phys. Rev. D 49, 2233 (1994); 49, 3352 (1994); D 50, 2225 (1994); D 59, 094002 (1999); J. Jalilian-Marian, A. Kovner, L. McLerran and H. Weigert, Phys. Rev. D 55, 5414 (1997); Yu. V. Kovchegov, Phys. Rev. D 54, 5463 (1996).

[10] A. Kovner, L. McLerran and H. Weigert, Phys. Rev. D 52, 3809 (1995); D 52, 6231 (1995).

[11] Yu. V. Kovchegov and D. H. Rischke, Phys. Rev. C 56, 1084 (1997).

[12] A. Krasnitz and R. Venugopalan, Nucl. Phys. B 557, 237 (1999); Phys. Rev. Lett. 84, 4309 (2000); ibid. 86, 1717 (2001); A. Krasnitz, Y. Nara and R. Venugopalan, Phys. Rev. Lett. 87, $192302(2001)$.

[13] L.V. Gribov, E.M. Levin, M.G. Ryskin, Phys. Rept. 100, 1 (1983).

[14] M. Gyulassy and L. McLerran, Phys. Rev. C 56, 2219 (1997).

[15] A. H. Mueller, Nucl. Phys. B 572, 227 (2000).

[16] D. Kharzeev and M. Nardi, Phys. Lett. B 507, 121 (2001).

[17] D. Kharzeev and E. Levin nucl-th/0108006.

[18] L. McLerran and J. Schaffner-Bielich, hep-ph/0101133; J. Schaffner-Bielich, D. Kharzeev, L. D. McLerran and R. Venugopalan, nucl-th/0108048.

[19] In general, one will obtain a non-zero contribution to $v_{2}\left(p_{t}\right)$ in the classical approach as long as a) the nuclei are finite and b) anisotropic.

[20] A. Krasnitz, Y. Nara and R. Venugopalan, to be published.

[21] Y. V. Kovchegov and K. Tuchin, hep-ph/0203213. 